\title{
21 Tesla MRI Microimaging of Rat Heart by Superparamagnetic Antimyoglobin Bound Nanoparticles R. SHARMA ${ }^{1,2}$
}

${ }^{1}$ National High Magnet Field Lab, Florida State University, Tallahassee, Florida, United States, ${ }^{2}$ Electrical Engg, FAMU-FSU College of Engineering, Tallahassee, Florida, United States

\begin{abstract}
$\underline{\text { Abstract }}$
Superparamagnetic iron-oxide bound antimyoglobin (SPIOM) characteristics were analyzed at different $\mathrm{pH}$. After injecting SPIOM in heart, ex vivo magnetic resonance microimaging (MRM) technique was used to visualize microvasculature of rat heart including cardiac arteries, veins, cordate tendons attached with valves. The measurement accuracy of ventricles, aorta, vasculature and dimensions of cordate tendons were close to coregistered histology measurements.

$\underline{\text { Object }}$
\end{abstract}

To visualize the rat heart tissue by 21 Tesla MRM to develop 3D atlas and compare MRM images with histology digital images.

$\underline{\text { Introduction }}$

Superparamagentic iron-oxide bound antimyoglobin (SPIOM) particles visualize myoglobin in rat heart tissue. Myoglobin plays significant role in myocardium and cardiac tissue as oxygen carrier. The binding of iron-oxide particles with myoglobin was pH dependant [1]. In presence of high magnetic field, iron-oxide particles exhibit dephasing effect that generates the MRM contrast [2]. Recently, 21 Tesla MRM at National High Magnetic Field Laboratory has enhanced the possibility of 3D visualization of rat heart aorta, ventricles, coronary tissue, myocardium, valves, cordate tendons and cardiac vasculature [2]. Superparamagnetic particles act as smart biosensors to localize molecules or molecular defects. For antimyoglobin in SPIOM, complementary clones of anti-human cardiac myoglobin IgG and primary antibodies (G-125-C) polyclonal antibodies in goat were used with the secondary myoglobin antibodies (A27370228P).

Materials and Methods

Using polyethylene as iron-oxide $\left(\gamma \mathrm{Fe}_{2} \mathrm{O}_{3}\right)$ encapsulating material bound with antimyoglobin in the presence of avidin-biotin complex, SPIOM particles were made available [3]. The $30 \mathrm{~nm}$ sized SPIOM was injected in rat femoral vein i.v. Soon after 4 minutes, rat heart was imaged at 11.7 Tesla for gross imaging and later excised rat heart was imaged at 21 Tesla using 3D FLASH, 3D GEFC and multislice multiecho techniques on PARAVISION 3.2 techniques (Bruker Biospin, Bellrica). Excised rat heart was processed for histology sections to coregister digital images with MRM images to generate rat heart atlas. Cardiac ventricles, aorta, auricles, coronary and blood vessels were measured by area and volumes using rendering and 3D reconstruction methods.

Results

The SPIOM particles were elongated and spherical in shape, 10-30 nm in diameter by TEM (Figure 1). The T1 weighted MR signal intensity of phantom SPIOM particles in capillary was high at $\mathrm{pH} 7.4$ than it was lower at $\mathrm{pH} 4.0$ due to myoglobin protein folding characteristics. Due to dephasing effect of SPIOM, the locations of nanoparticles deposits showed up as signal loss. Whole animal images showed the localized SPIOM distribution around cardiac surfaces shown in Figures 2 and 3 . The excised heart tissue showed rat heart ventricles, auricles and coronary territories were distinct at 21 Tesla (Figure 4). Main features of 21 Tesla MRM were: 1.the signalnoise-ratio and resolution were high with spatial resolution of 20 microns; 2. 3D FLASH images were acquired in 12 seconds; 3. 3D GEFC images showed noise on background but acquisition time in less than 15 seconds;4. MSME images showed better structural details; 5.the 3D reconstruction showed distinct boundaries, areas and vasculature; 6. main features of heart right and left chambers, septum, tricuspid and mitral valves were measurable; 7 . cordate tendons (shown as arrows in Figure 4) were distinct as attached with cusps. By histological match, MRI measured real dimensions while histology processing reduced the tissue by $20-25 \%$ due to shrinkage. The volume rendering generated 3D atlas based on 3D segmentation and orientation of cardiac vasculature and chambers. However, the limitations of 21 Tesla MRM were observed for long time experiments due to less stable gradients, coil Bo inhomogeneity and noise as main determinants during 3D experiments.



Discussion

The ultrahigh resolution MRM is new research tool with lot of promise in superior spatial resolution up to 20 micron level visualization of microvasculature in rat heart tissue in both in vivo or ex vivo experiments. By use of superparamagnetic iron oxide particles, nanotechnology further provides smart biosensor approach using them for MRM. Myoglobin has benefit of oxygen carrying and supply cardiac muscles for their continuous function. So, SPIOM is ideal biosensor and imaging contrast agent. 3D visualization is other benefit of 21 Tesla MRM experiments. However, ultrahigh magnetic field has own demerits which are not fully known. Our experience showed frequently that SNR and noise were highly dependent on gradient stability, $B_{0}$ inhomogeneity and susceptibility effects. Other major artifact was shrinkage of tissue during histology processing. Despite of these limitations, 3D visualization with microlevel accuracy is main benefit.

Power of 21 Tesla MR Microscopy unlike before:

- Ultrahigh Structural \& Anatomic Resolution

- Cardiac muscle fiber orientation,

- Dimensionality of dynamic cardiac motion

- Localization of cardiac muscle protein specific nanoparticles as molecular signatures 


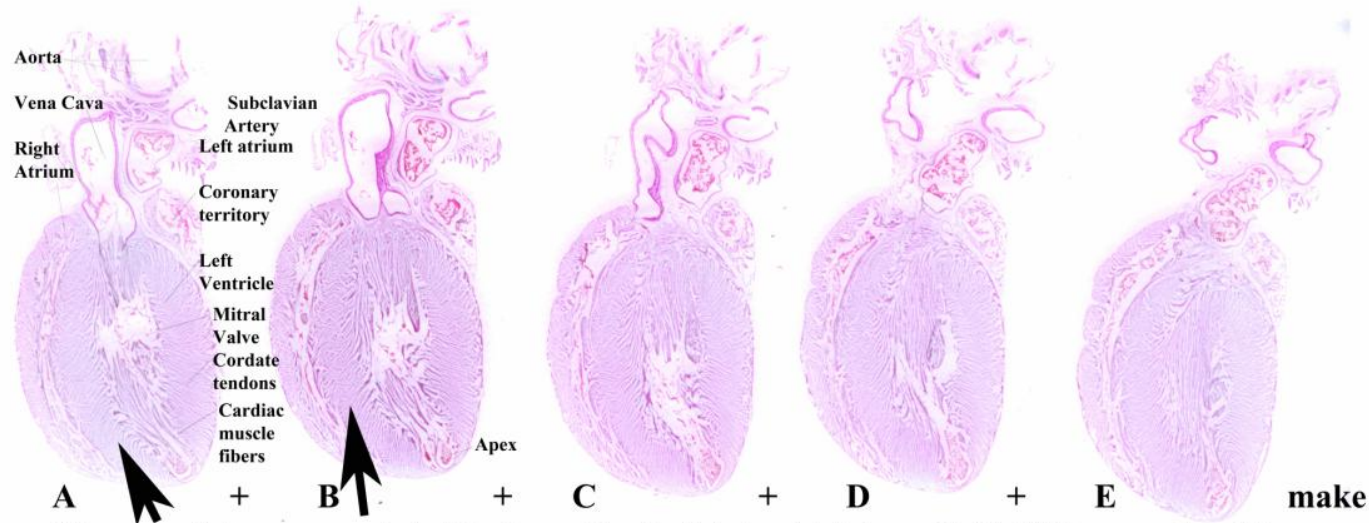

Nanoparticles accumulated in tissue(dark stain) histology digital 3D reconstruct image

Conclusion

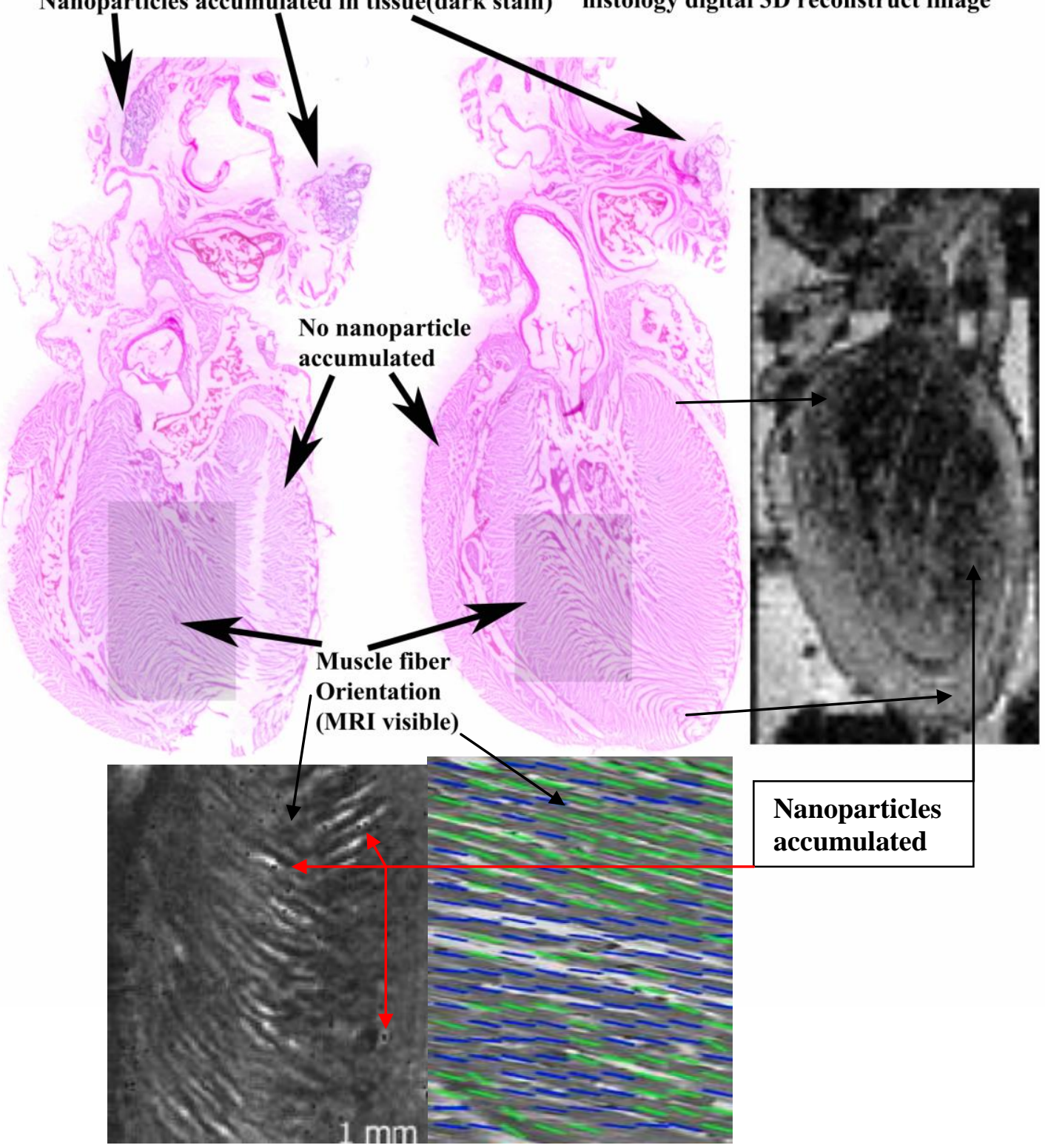

Superparamagnetic particles enhanced the contrast. At 21 Tesla, the rat heart showed better resolution and high SNR with distinct cardiac morphology and vasculature. The histology matched MRM images showed the power of microimaging to predict the microdetails of different cardiac territories. The enhanced ultrahigh resolution microimaging may be useful molecular imaging and functional monitoring research tool.

$\underline{\text { References }}$

1. Sharma R, Haik Y, Chen CJ J Expt Nano Sci. 2006,1 \& NTSI 2006 \# 905

2. Sharma R, Kwon S J Expt Nano Sci 2006,1 \& NTSI 2006 \# 711

3. Chatterjee J, Haik Y, Chen CJ 2006 Polymeric encapsulation of nanoparticles. US Patent \# 7081489 